This item was submitted to Loughborough's Research Repository by the author.

Items in Figshare are protected by copyright, with all rights reserved, unless otherwise indicated.

\title{
The influence of the electronic structure method on intersystem crossing dynamics. The case of thioformaldehyde
}

PLEASE CITE THE PUBLISHED VERSION

https://doi.org/10.1021/acs.jctc.9b00282

\section{PUBLISHER}

(c) American Chemical Society (ACS)

\section{VERSION}

AM (Accepted Manuscript)

\section{PUBLISHER STATEMENT}

This document is the Accepted Manuscript version of a Published Work that appeared in final form in Journal of Chemical Theory and Computation, copyright @ American Chemical Society after peer review and technical editing by the publisher. To access the final edited and published work see https://pubs.acs.org/doi/10.1021/acs.jctc.9b00282.

\section{LICENCE}

CC BY-NC-ND 4.0

\section{REPOSITORY RECORD}

Mai, Sebastian, Andrew J. Atkins, Felix Plasser, and Leticia Gonzalez. 2019. "The Influence of the Electronic Structure Method on Intersystem Crossing Dynamics. The Case of Thioformaldehyde". Loughborough University. https://hdl.handle.net/2134/37771. 


\title{
The Influence of the Electronic Structure
} Method on Intersystem Crossing Dynamics. The Case of Thioformaldehyde

\author{
Sebastian Mai, ${ }^{\dagger}$ Andrew J. Atkins, ${ }^{\dagger}$, Felix Plasser, ${ }^{\dagger, \ddagger}$ and Leticia González, ${ }^{* \dagger}$ \\ $\dagger$ Institute of Theoretical Chemistry, Faculty of Chemistry, University of Vienna, \\ Währinger Str. 17, 1090 Vienna, Austria \\ $\ddagger$ Present address: Department of Chemistry, Loughborough University, Loughborough, LE11 \\ 3TU, UK. \\ T These authors contributed equally to this work. \\ E-mail: leticia.gonzalez@univie.ac.at
}

\begin{abstract}
The ability of different electronic structure methods to describe correctly intersystem crossing dynamics is evaluated, using thioformaldehyde as a test case. Mischievously, all methods considered - ranging from the multi-reference methods MRCISD, MS-CASPT2, or SA-CASSCF, to the single-reference methods ADC(2), CC2, and TDDFT in different flavours - provide the same state ordering and energies of the low-lying singlet and triplet electronic excited states within an acceptable error of $0.2-0.3 \mathrm{eV}$. However, the outcome of the non-adiabatic simulations after excitation to the lowest $S_{1}\left({ }^{1} n \pi^{*}\right)$ state are dramatically different. While MS-CASPT2, ADC(2), BP86, and PBE do not transfer population to the triplet states within 500 fs - in consonance with experimental evidence-SA-CASSCF, B3LYP, and BHHLYP predict intersystem crossing yields between $3 \%$ and $21 \%$ within the same time. The different
\end{abstract}


excited state dynamics can be rationalized by inspecting potential energy profiles along the $\mathrm{C}-\mathrm{S}$ bond stretch mode and single-triplet energy gaps. It is found that already at a C-S bond length of $1.9 \AA$, all the single-reference methods struggle to describe the correct asymptotic behavior of the potentials. Moreover, some methods, including SACASSCF, obtain incorrect ${ }^{1} n \pi^{*}-{ }^{3} \pi \pi^{*}$ energy gaps, leading to compensation of errors (ADC(2), BP86, PBE), or wrong dynamics (SA-CASSCF, B3LYP, BHHLYP). Only the accurate MRCISD and MS-CASPT2 methods are able to describe the $\mathrm{C}-\mathrm{S}$ bond correctly and thus able to deliver the correct potential energy surfaces and dynamics for the right reason. A correlation with the amount of Hartree-Fock exchange in the density functional and the easiness to access the ${ }^{3} \pi \pi^{*}$ state from the ${ }^{1} n \pi^{*}$ is able to explain the different behavior observed for GGA and hybrid functionals. It is thus illustrated that even in the case of a simple molecule, like $\mathrm{CH}_{2} \mathrm{~S}$, the sole assessment of vertical excitation energies as reliability predictors for non-adiabatic is inadequate. The reason is that ISC does not occur at the FC geometry, but rather at distorted geometries where the singlet-triplet gaps become small. Hence, a characterization of the potential energy surfaces beyond the Franck-Condon region is mandatory.

\section{Introduction}

Intersystem crossing (ISC) is the radiationless transition between electronic states of different spin multiplicities, e.g., from a singlet to a triplet state. Given the involvement of ISC

in many applications such as dye-sensitized solar cells, ${ }^{1}$ organic light-emitting diodes, ${ }^{2,3}$ or photodynamic therapy, ${ }^{4,5}$ many studies aim at understanding this oftentimes highly complex process. ${ }^{6}$ Only recently it has become possible to carry out nonadiabatic molecular dynamics simulations including ISC and internal conversion on the same footing. ${ }^{7-12}$

A key ingredient in any nonadiabatic molecular dynamics simulation is the level of theory employed to calculate the electronic potential energy surfaces (PESs), on which the nuclei are moving. The aim of this work is to compare the effect that different electronic structure 
methods have on such simulations. An important question is to investigate how reliable it is to validate these methods only at the Franck-Condon (FC) geometry, and assume that beyond that geometry the PESs will be parallel at different levels of theory, an issue that, for singlet PESs, has been the focus of several previous studies, e.g., Refs. ${ }^{13-16}$ With this in mind, here we compare the accuracy of different electronic structure methods to describe the ISC dynamics of thioformaldehyde $\left(\mathrm{CH}_{2} \mathrm{~S}\right)$.

$\mathrm{CH}_{2} \mathrm{~S}$ has been chosen because it is small enough to enable high-level benchmark calculations, to which cheaper levels of theory can be compared, in terms of total ISC yields, bond length variations, and other parameters. From the experimental side, the low-lying electronic excited states of thioformaldehyde have been studied in detail for several decades. ${ }^{17-20}$ Thioformaldehyde possesses two low-lying $n \pi^{*}$ states, a singlet (origin at $2.03 \mathrm{eV}$ ) and a triplet (at $1.80 \mathrm{eV}$ ). ${ }^{19}$ The second triplet state, ${ }^{3} \pi \pi^{*}$, is located around $2.70 \mathrm{eV} .{ }^{21}$ After excitation

to the ${ }^{1} n \pi^{*}$ state, fluorescence with a life time of $70-300 \mu \mathrm{s}^{19,22}$ is typically reported. The analysis of the rovibrational structure of the absorption spectrum revealed that many lines are perturbed by a number of singlet-triplet interactions. The ISC rate is not reported to our knowledge; based on the high fluorescence emission yields, ${ }^{19,22,23}$ it is likely to be small. For comparison, an ISC time scale of around 400 ps was obtained in surface hopping simulations for formaldehyde, ${ }^{24}$ so it can be expected that thioformaldehyde exhibits a time constant around a few hundred picoseconds.

\section{Computational Details}

\subsection{Electronic Structure Methods}

The state-averaged complete active space self consistent field (SA-CASSCF), ${ }^{38,62}$ multistate complete active space second order perturbation theory (MS-CASPT2), ${ }^{31-34,63}$ multireference configuration interaction including single and double excitations with the Pople size-extensivity correction $(\mathrm{MRCISD}+\mathrm{P}),{ }^{27,28,64}$ time-dependent density functional theory 
Table 1: Electronic structure levels of theory included in the present study. The column "Dynamics" indicates which levels of theory were employed to simulate nonadiabatic dynamics.

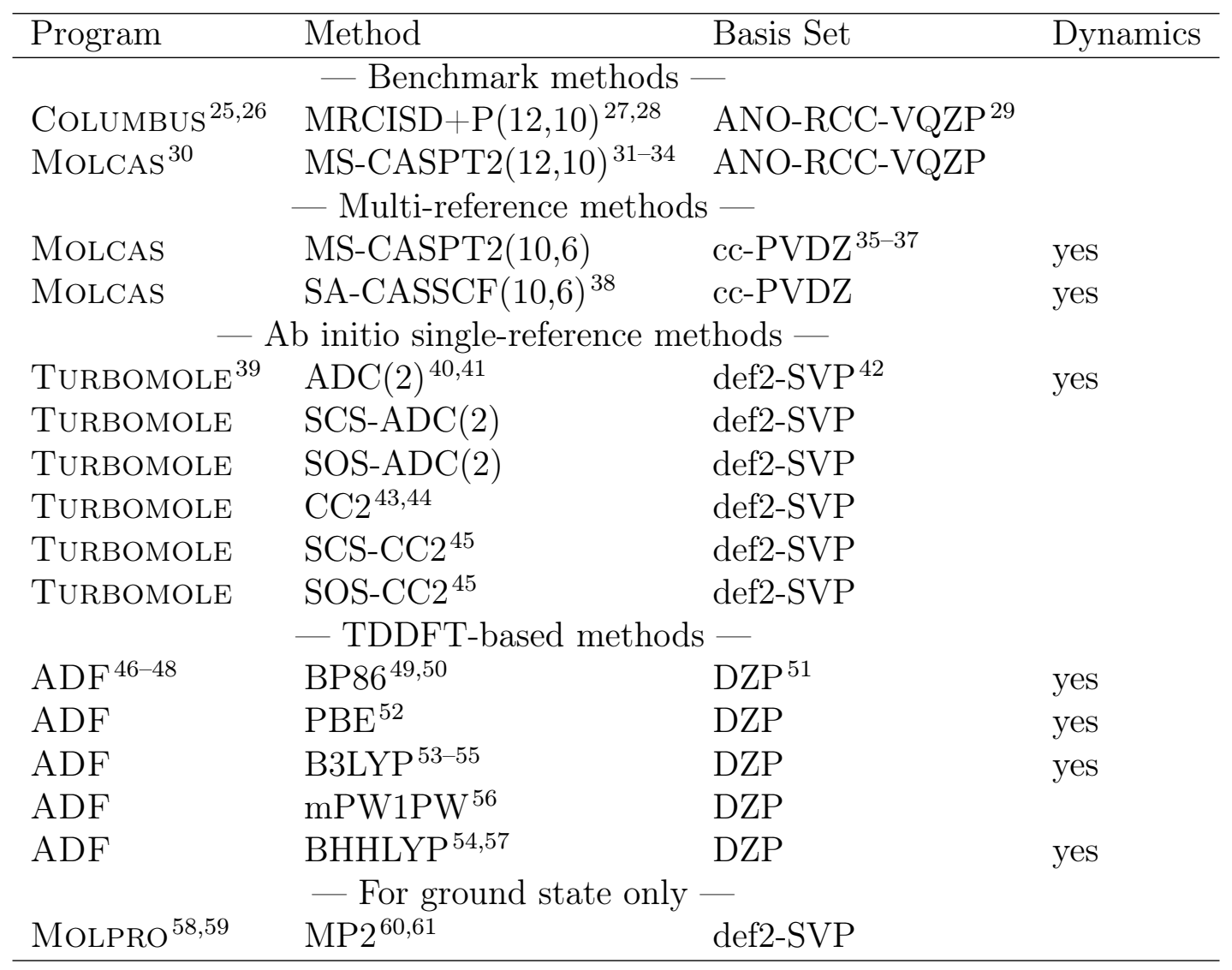

(TDDFT) ${ }^{65,66}$ and algebraic diagrammatic construction scheme for the polarization propagator to the second order $(\operatorname{ADC}(2))^{40,41,67}$ methods are employed to investigate the photophysics of $\mathrm{CH}_{2} \mathrm{~S}$. Table 1 compiles the employed quantum chemistry codes, methods, the basis sets, and relevant references.

For the most accurate multi-reference calculations (MRCISD+P, MS-CASPT2, and SACASSCF), the active space orbitals are presented in Figure S1 in the supporting information (SI). The MRCISD $+\mathrm{P}$ computations utilized orbitals coming from a CASSCF $(12,10)$ computation with state-averaging including 2 singlet and 2 triplet states (i.e., $\mathrm{SA}(2 \mathrm{~S}+2 \mathrm{~T})$ CASSCF $(12,10))$, and were performed within $C_{2 v}$ symmetry, with 6 frozen core orbitals, and with the Douglas-Kroll-Hess scalar relativistic Hamiltonian. ${ }^{68}$ The SA-CASSCF and 
MS-CASPT2 calculations were also performed using the Douglas-Kroll-Hess Hamiltonian. Following the suggestions in Ref., ${ }^{69}$ the MS-CASPT2 computations with the large ANORCC-VQZP basis set used an IPEA shift ${ }^{70}$ of 0.25 a.u. and an imaginary level shift ${ }^{71}$ of 0.2 a.u.; the MS-CASPT2 computations with the cc-pVDZ used an IPEA shift of zero and an imaginary level shift of 0.3 a.u. The $\mathrm{ADC}(2)$ and $\mathrm{CC} 2$ computations used the resolution-of-the-identity approximation ${ }^{72}$ and a non-relativistic Hamiltonian. All TDDFT calculations utilized the scalar-relativistic zeroth order regular approximation (ZORA), ${ }^{73-77}$ with numerical integration performed with a Becke grid 78,79 of "good" quality and a Zlm fit ${ }^{80}$ of "normal" quality. All TDDFT calculations used the Tamm-Dancoff approximation (TDA) ${ }^{81,82}$ and the adiabatic local density approximation (ALDA). ${ }^{83}$ To test the influence of the exchange-correlation functional, we considered two GGA functionals (BP86, ${ }^{49} \mathrm{PBE}^{52}$ ), two typical hybrid functionals (B3LYP, ${ }^{53} \mathrm{mPW} 1 \mathrm{PW}{ }^{56}$ ), and one hybrid with a large fraction of Hartree-Fock (HF) exchange (BHHLYP $\left.{ }^{57}\right)$.

The MRCISD $+\mathrm{P}(12,10)$ and MS-CASPT2(12,10) computations with the ANO-RCCVQZP basis set are the most accurate ones. They serve as reference methods here and will be denoted as "benchmark" methods.

The ground state geometry - which is employed for the vertical excitation calculations, the starting point of the PES scans, and the initial condition generation (see below)was optimized with a smaller set of electronic structure methods. The MRCISD $+\mathrm{P}, \mathrm{MS}-$ CASPT2, and ADC(2)/CC2 methods all shared the ground state geometry optimized at the MP2/def2-SVP level of theory. For the SA-CASSCF method, the ground state geometry was obtained at the same SA-CASSCF level of theory. All TDDFT methods were used with the same ground state geometry computed at the BP86/DZP level.

Using these geometries, we performed vertical excitation calculations, computing the lowest excited singlet state $\left({ }^{1} n \pi^{*}\right)$ and the two lowest triplet states $\left({ }^{3} n \pi^{*}\right.$ and $\left.{ }^{3} \pi \pi^{*}\right)$. The same states were included in the PES scan along the C-S bond from the initial, optimized value up to $2.2 \AA$, with all other internal coordinates left unchanged. Along each of the PES 
scans, we also computed the SOC matrix elements, as given in the relevant literature for Columbus,${ }^{84,85}$ Molcas, ${ }^{86}$ Turbomole/Orca,${ }^{67,87}$ and ADF. ${ }^{88,89}$

\subsection{Nonadiabatic Dynamics Simulations}

For the ab initio excited-state dynamics simulations, we used the Surface Hopping including ARbitrary Couplings (SHARC) program package ${ }^{7-9}$ in combination with a subset of electronic structure methods-MS-CASPT2(10,6), SA-CASSCF(10,6), ADC(2), and TDDFT with two GGA (BP86, PBE) and two hybrid (B3LYP, BHHLYP) exchange-correlation functionals. The initial conditions for the dynamics were randomly sampled from ground-state harmonic oscillator Wigner distributions,${ }^{90,91}$ based on frequency calculations for the optimized geometries as specified above. For each electronic structure method, we generated 1000 initial conditions and computed an absorption spectrum with the line-broadening method. ${ }^{92}$

Subsequently, for each method we excited 200 initial conditions to the $S_{1}$ state, and propagated 200 trajectories for 1000 fs using a time step of $0.5 \mathrm{fs}$. The electronic wave functions were propagated with a 0.02 fs time step with the local diabatization method. ${ }^{93}$ The required (approximate) wave function overlaps were computed for all methods with the WFoverlap code; ${ }^{94}$ to this end, for SA-CASSCF we used the actual wave function,

for MS-CASPT2 the perturbatively-modified ${ }^{33}$ CASSCF wave function, and approximate configuration interaction singles (CIS)-like wave functions for $\mathrm{ADC}(2)^{95}$ and TDDFT. ${ }^{96,97} \mathrm{An}$ energy-based decoherence correction ${ }^{98}$ was applied to the electronic wave function coefficients in the eigenfunction basis. ${ }^{9}$ SOCs were computed on-the-fly at the same level of theory as energies and gradients. In all cases, the trajectories considered the $S_{0}, S_{1}, T_{1}$, and $T_{2}$, giving a total of 8 spin-coupled states, as the triplet components are treated separately. ${ }^{8}$ 


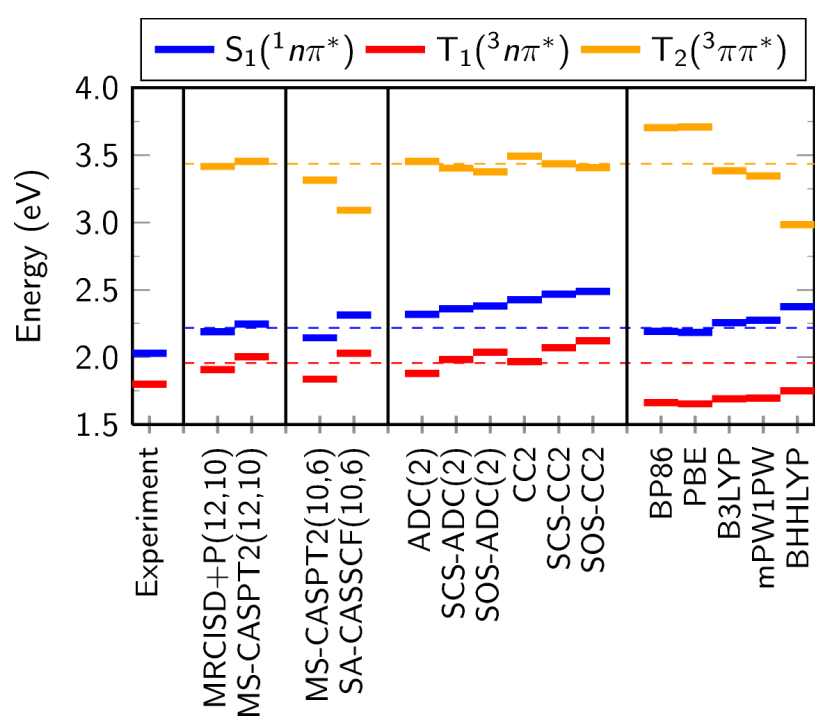

Figure 1: Vertical excitation energies at the Franck-Condon geometry obtained with the different electronic structure methods as indicated. The dashed lines show the average between the results of MRCISD $+\mathrm{P}$ and MS-CASPT2 $(12,10)$.

\section{Results}

\subsection{Vertical Excitation Energies}

The most straightforward way of comparing the performance of the electronic structure methods is to inspect the vertical excitation energies at the FC geometry. These energies are sketched in Figure 1 and listed in Table 2. All electronic structure methods agree on the state characters, with $S_{1}$ and $T_{1}$ being $n \pi^{*}$ and $T_{2}$ being $\pi \pi^{*}$ (see Figure $\mathrm{S} 2$ in the SI).

Figure 1 also shows experimental ${ }^{19} 0-0$ transition energies for the two lowest states. These serve as a reference but as they are not directly comparable to the computed vertical energies, the two benchmark calculations (MRCISD $+\mathrm{P}$ and MS-CASPT2 $(12,10)$ ) will be used to compare with the rest of the methods. In any case, these two calculations provide results in remarkably good agreement with the experiment. Thus, the average of the MR$\mathrm{CISD}+\mathrm{P}$ and MS-CASPT2 $(12,10)$ excitation energies, which are indicated in the figure as dashed lines, will be used for further comparisons.

The differences between the results are typical for such a diverse set of electronic structure

methods, in the sense that the excitation energies agree within $0.2-0.3 \mathrm{eV} .{ }^{99}$ Compared 
Table 2: Vertical excitation energies for the different electronic structure methods, for the $S_{1}, T_{1}$, and $T_{2}$ excited states. The two last columns give the RMSD of the excitation energies $(E)$ and the $S_{1}-T_{1}, S_{1}-T_{2}$, and $T_{1}-T_{2}$ energy differences $(\Delta E)$, relative to the average of the two reference methods.

\begin{tabular}{lccccc}
\hline Method & $S_{1}$ & $T_{1}$ & $T_{2}$ & RMSD & RMSD \\
& $\left({ }^{1} n \pi^{*}\right)$ & $\left({ }^{3} n \pi^{*}\right)$ & $\left({ }^{3} \pi \pi^{*}\right)$ & $(E)$ & $(\Delta E)$ \\
\hline Exp $^{19}$ & 2.03 & 1.80 & & & \\
MRCISD+P(12,10) & 2.19 & 1.91 & 3.42 & & \\
MS-CASPT2 $(12,10)$ & 2.25 & 2.00 & 3.45 & & \\
MS-CASPT2(10,6) & 2.14 & 1.84 & 3.31 & 0.11 & 0.04 \\
SA-CASSCF $(10,6)$ & 2.31 & 2.03 & 3.09 & 0.21 & 0.35 \\
ADC(2) & 2.32 & 1.88 & 3.45 & 0.07 & 0.13 \\
SCS-ADC(2) & 2.36 & 1.98 & 3.40 & 0.09 & 0.13 \\
SOS-ADC(2) & 2.38 & 2.04 & 3.38 & 0.11 & 0.16 \\
CC2 & 2.43 & 1.97 & 3.49 & 0.13 & 0.15 \\
SCS-CC2 & 2.47 & 2.07 & 3.44 & 0.16 & 0.18 \\
SOS-CC2 & 2.49 & 2.12 & 3.41 & 0.18 & 0.21 \\
BP86 & 2.19 & 1.66 & 3.70 & 0.23 & 0.40 \\
PBE & 2.18 & 1.65 & 3.71 & 0.24 & 0.41 \\
B3LYP & 2.26 & 1.69 & 3.38 & 0.16 & 0.22 \\
mPW1PW & 2.28 & 1.70 & 3.34 & 0.16 & 0.22 \\
BHHLYP & 2.37 & 1.75 & 2.99 & 0.30 & 0.43 \\
\hline
\end{tabular}

to the two reference methods, the best result is obtained with the smaller MS-CASPT2 computation, whose energies are shifted by about $0.1 \mathrm{eV}$ from the reference, whereas the energy gaps are accurate to $0.04 \mathrm{eV}$, as measured by the root-mean-square deviation (RMSD). SA-CASSCF delivers accurate results only for the $n \pi^{*}$ states $\left(S_{1}\right.$ and $\left.T_{1}\right)$, whereas the ${ }^{3} \pi \pi^{*}$ state is significantly underestimated. The different flavors of $\mathrm{ADC}(2)$ and $\mathrm{CC} 2$ all provide very good triplet energies, but systematically overestimate the $S_{1}$. Still, these methods provide RMSDs of $0.07-0.18 \mathrm{eV}$ and can be regarded as accurate. Within the TDDFT methods, the hybrid functionals (B3LYP and mPW1PW) appear to perform best, although all TDDFT methods generally underestimate the $T_{1}$ energy. Furthermore, the energy of the $T_{2}$ state depends strongly on the amount of Hartree-Fock exchange, which leads to large RMSDs for the GGA functionals and BHHLYP. We also note that the two GGAs provide virtually identical results; the same holds for B3LYP and mPW1PW. We note here that all TDDFT methods employed the TDA ${ }^{81,82}$ and ALDA. ${ }^{83}$ As shown in Table S1 in the 
SI, usage of the TDA is imperative for a reasonable description of the $T_{2}$ state, because that state suffers from triplet instability problems ${ }^{100}$ otherwise. Table S1 also shows that not employing the ALDA tends to produce slightly better results, but unfortunately in ADF gradient calculations cannot be performed without the ALDA, making dynamics simulations not possible.

A very important ingredient for ISC dynamics is the energy difference between the singlet and triplet states, according to the energy-gap law. ${ }^{101}$ Experimentally, the $S_{1}-T_{1}$ energy gap $(0-0)$ was estimated to be $0.23 \mathrm{eV} .{ }^{102}$ This value is closely reproduced by the benchmark methods $(0.28$ for MRCISD $+\mathrm{P}, 0.24$ for MS-CASPT2 $(12,10))$ and the other multi-reference methods, but overestimated by all other calculations. However, the SOC matrix elements between $S_{1}\left({ }^{1} n \pi^{*}\right)$ and $T_{1}\left({ }^{3} n \pi^{*}\right)$ are symmetry forbidden within the $C_{2 v}$ point group. Consequently, ISC might preferably take place between $S_{1}\left({ }^{1} n \pi^{*}\right)$ and $T_{2}\left({ }^{3} \pi \pi^{*}\right)$, which makes the $S_{1}-T_{2}$ gap more important to assess. For this gap, the benchmark computations give a value of around $1.2 \mathrm{eV}$. SA-CASSCF, CC2, and BHHLYP significantly underestimate this value, while BP86 and PBE give a too large gap.

In summary, all electronic structure methods provide excitation energies within less than $\pm 0.3 \mathrm{eV}$ from the benchmark calculations and give qualitatively the same state ordering. It could then be expected that MS-CASPT2 $(10,6), \mathrm{ADC}(2) / \mathrm{CC} 2$, and B3LYP/mPW1PW will provide a dynamics closest to that of the benchmark methods, while SA-CASSCF, BP86/PBE, and BHHLYP might not be completely accurate due to their error in the singlettriplet gaps. Nevertheless, based only on these vertical energy results it is difficult to predict the outcome of the dynamics.

\subsection{Nonadiabatic Dynamics Simulations}

Excited-state dynamics simulations were performed for seven of the 15 electronic structure methods considered so far. We did not perform dynamics with the two benchmark methods $(\mathrm{MRCISD}+\mathrm{P}(12,10)$ and MS-CASPT2 $(12,10))$, due to their computational expense. How- 


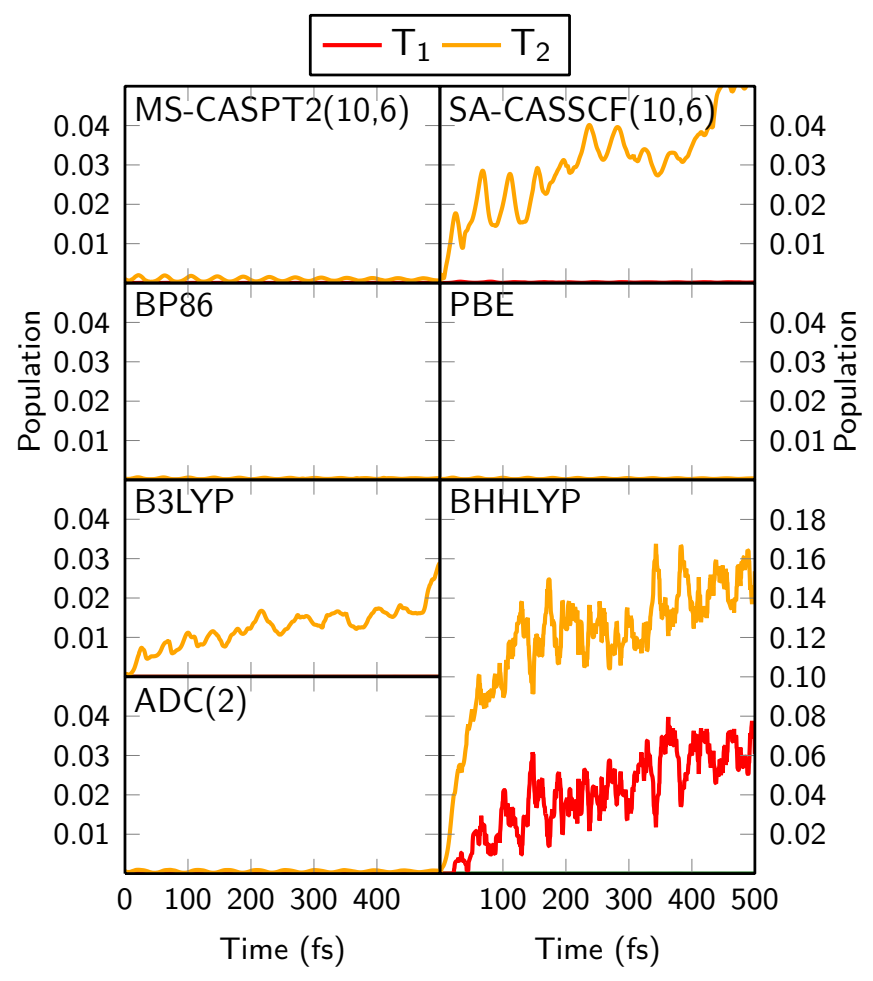

Figure 2: Populations of 200 trajectories for a duration of $500 \mathrm{fs}$ of the $\mathrm{T}_{1}$ (red) and $\mathrm{T}_{2}$ (orange) states in $\mathrm{CH}_{2} \mathrm{~S}$ using different levels of theory. The populations shown here are the sum of the squared coefficients for each state of the wave function set rather than the 'classical' populations.

ever, as MS-CASPT2(10,6) delivers very similar stationary results (see also later) as the benchmark methods, we will employ the latter method as the reference for comparison.

Besides MS-CASPT2(10,6), the nonadiabatic dynamics simulations were carried out with SA-CASSCF $(10,6), \operatorname{ADC}(2)$, and TDDFT with the four functionals BP86, PBE, B3LYP, and BHHLYP (as indicated in Table 1). The aim of the dynamics simulations is to find whether the vertical excitation energies allow estimating the suitability of the different electronic structure methods.

For all 7 methods considered, Figure 2 shows the time evolution of the populations, based on 200 trajectories and 500 fs simulation time. The populations shown are the sums of the squared state coefficients in the MCH basis. ${ }^{9}$

We first analyze the dynamics employing MS-CASPT2(10,6) potentials. As can be seen in Figure 2, at this level of theory no persistent triplet population is building up within 
500 fs. Consequently, no trajectory performed any hop out of the dominantly singlet state into a dominantly triplet state. The same behavior was found when the simulation time was extended to 1000 fs (not shown). The population plot only shows small, damped oscillations of the $T_{2}$ population between zero and 0.002 . These oscillations stem from the fact that with SHARC the trajectories are propagated on spin-mixed potential energy surfaces (the so-called "diagonal" representation" ${ }^{9}$ ), and the composition of the spin-mixed active potential changes between $100 \%$ and $99.8 \%$ singlet character. The reason for this variation in spin mixing lies in the variation of the $\mathrm{C}-\mathrm{S}$ bond length over time, which is shown in Figure 3 for all electronic structure methods. As can be seen, the variation of the bond length matches accurately with the apparent $T_{2}$ population. The variation in the C-S bond length modulates the $S_{1}-T_{2}$ energy gap (see below), which in turn has a direct effect on the amount of spin mixing. The oscillations in the $T_{2}$ population can be regarded as the SHARC equivalent of perturbation of the vibrational levels by the singlet-triplet interaction, as singlet-triplet mixing in SHARC also has an effect on the gradients and thus the mixing can affect the vibrational motion.

Another observation in Figure 3 is the coherence of the C-S bond stretch motion over all 200 trajectories. The oscillations are visibly coherent for the full 500 fs time window, although coherence slowly degrades over time due to coupling to the other vibrational degrees of freedom. The oscillation period is approximately $43 \mathrm{fs}$ for the MS-CASPT2 trajectories, corresponding to $775 \mathrm{~cm}^{-1}$ and in good agreement with the experimental findings (41 fs or $\left.820 \mathrm{~cm}^{-1}\right) .19$

Based on the MS-CASPT2 results, we conclude that $\mathrm{CH}_{2} \mathrm{~S}$ does not undergo ISC on a sub-ps time scale after vertical excitation from the ground state minimum. This is fully consistent with experimental findings on the molecule, ${ }^{19,22,23}$ and with 300 ps surface hopping simulations on the related formaldehyde molecule. ${ }^{24}$ In the following, we will discuss which electronic structure methods are able to reproduce these findings.

Next we consider the SA-CASSCF dynamics, which was approximately 10 times cheaper 


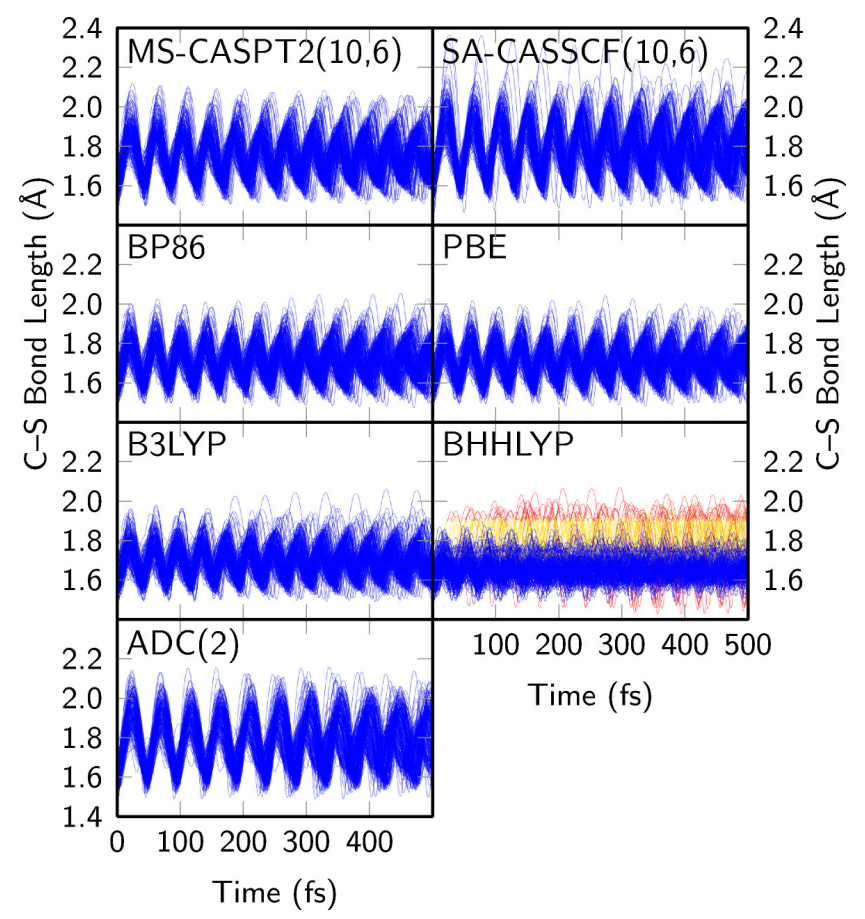

Figure 3: C-S bond length variation over the first 500 fs for all 200 trajectories calculated using the indicated method. For BHHLYP, color indicates the state in which the trajectory is currently moving $\left(S_{1}\right.$ blue, $T_{1}$ red, $T_{2}$ yellow).

than the MS-CASPT2 dynamics. However, SA-CASSCF does not produce the correct ISC dynamics. As can be seen in Figure 2, for this method a non-negligible fraction of the trajectories has the propensity to undergo ultrafast ISC to the $T_{2}\left({ }^{3} \pi \pi^{*}\right)$ state. Already after half of a C-S oscillation period (i.e., at around $25 \mathrm{fs}$ ) the $T_{2}$ population reaches almost $2 \%$, and each further oscillation increases the $T_{2}$ population further. Within 500 fs, the $T_{2}$ population reaches $5 \%$. This is consistent with the results of a related generalized ab initio multiple spawning (GAIMS) study. ${ }^{103}$ GAIMS is a nonadiabatic dynamics method that solves the time-dependent Schrödinger equation in a basis of classically moving, frozen Gaussians, effectively adding some quantum-mechanical effects to surface hopping simulations. Using SA-CASSCF $(4,3) / 6-31 \mathrm{G}^{*}$, the GAIMS method predicted a $T_{2}$ population of $5 \%$ after already 200 fs (present work: 3\% after $200 \mathrm{fs}$ ). This shows that the too large ISC rate is not due to the SHARC formalism, but is directly related to the SA-CASSCF PESs.

Next we shall consider the GGA exchange-correlation functionals BP86 and PBE, which 
give virtually identical results, as seen in Figures 2 and 3. With either functional, no population transfer to the $T_{2}$ occurs in the first $500 \mathrm{fs}$. However, as discussed for the MS-CASPT2 trajectories, there are weak oscillations of the $T_{2}$ population due to the modulation of the C-S bond length and consequently of the $S_{1}-T_{2}$ energy gap and the singlet-triplet mixing. These oscillations are smaller by a factor of three, compared to the MS-CASPT2 results. This decreased $T_{2}$ contribution might be related to the very large $S_{1}-T_{2}$ energy gap predicted by the GGA functionals, as shown in Figure 1.

The situation is quite different in the case of the hybrid functional B3LYP. Similar to SACASSCF, B3LYP predicts a non-zero ISC yield in the simulations, reaching a $T_{2}$ population of $3 \%$ after $500 \mathrm{fs}$. The evolution of the $T_{2}$ population still shows an oscillatory pattern due to the influence of the C-S stretch mode.

The BHHLYP functional performs even worse in the ISC dynamics, as can be easily spotted in Figure 2. With BHHLYP, one observes a rapid population transfer from the $S_{1}$ to the triplet states (even requiring a different $y$ axis scale). Unlike in the other simulations, both $T_{2}$ and $T_{1}$ are populated here, indicating that at this level of theory the two states have an easily accessible crossing. Note that the figure shows adiabatic populations, i.e., where $T_{1}$ is the lower-energy state no matter the state character - hence, in the presence of a crossing the $T_{1}$ population is not equivalent to the ${ }^{3} n \pi^{*}$ population. The fast oscillations between $T_{1}$ and $T_{2}$ are then only related to surface switching at the $T_{1} / T_{2}$ crossing, but are not related to population transfer between ${ }^{3} n \pi^{*}$ and ${ }^{3} \pi \pi^{*}$. We estimated the ${ }^{3} n \pi^{*}$ population to be approximately half of the $T_{1}$ population shown in Figure 2, i.e., around $3 \%$ after 500 fs. The total triplet population after $500 \mathrm{fs}$ is $21 \%$ after $500 \mathrm{fs}$, seven times higher than with B3LYP even though the initial conditions were identical.

The final electronic structure method considered for the dynamics is $\mathrm{ADC}(2)$. The populations in Figure 2 show that no ISC occurs in this case, with the weak oscillations of $T_{2}$ having an amplitude about half as large as for MS-CASPT2. This is due to the overestimated $S_{1}-T_{2}$ energy gap that $\mathrm{ADC}(2)$ produces. The C-S stretch motion in Figure 3 is 
quite similar to the MS-CASPT2 results, indicating that the shape of the $S_{1}$ potential is well-described with $\operatorname{ADC}(2)$.

\subsection{Potential Energy Surfaces}

The widely varying results shown in the previous section - with ISC yields ranging from $0 \%$ to $20 \%$ - can hardly be explained from predictions based on the vertical excitation data in Table 2. The reason is that ISC does not occur at the FC geometry, but rather at distorted geometries where the singlet-triplet gaps become small. Hence, electronic structure methods for dynamics simulations should not only be validated from vertical excitation energies. Instead, it is essential that the electronic structure methods are scrutinized based on all relevant parts of the PESs, ${ }^{13-16}$ even though this makes comparison to experimental data more difficult.

For $\mathrm{CH}_{2} \mathrm{~S}$, the principal degree of freedom involved in the excited-state dynamics is the C-S bond stretch, ${ }^{103}$ which controls the energy difference between singlet and triplet states. At large bond lengths $S_{1}, T_{1}$, and $T_{2}$ approach each other as the $\pi$ bond becomes weaker; the three states correspond to degenerate states of the dissociated system $\left(\mathrm{CH}_{2}+\mathrm{S}\right)$. Furthermore, the increased bond length increases the magnitude of SOCs between the states. Thus, in Figure 4 we show PES scans along the C-S bond stretch coordinate for all 15 electronic structure methods considered here, together with the positions of the excited-state minima. The numeric data for the minima is given in Table S2 in the SI.

As in the case of the vertical excitation calculations, the two benchmark methods agree very well with each other, both qualitatively and quantitatively. After downshifting the MS-CASPT2(12,10) PESs by $0.06 \mathrm{eV}$, the remaining RMSD (over all four states and across the full scan range) between MRCISD $+\mathrm{P}$ and MS-CASPT2 is only $0.05 \mathrm{eV}$. In general, the two scans show that upon elongation of the C-S bond the three excited states go through a minimum before increasing in energy at long bond lengths. The ${ }^{1} n \pi^{*}$ and ${ }^{3} n \pi^{*}$ approach each other quickly — with their gap falling below $0.1 \mathrm{eV}$ at $1.9 \AA$-reflecting a decay of the 


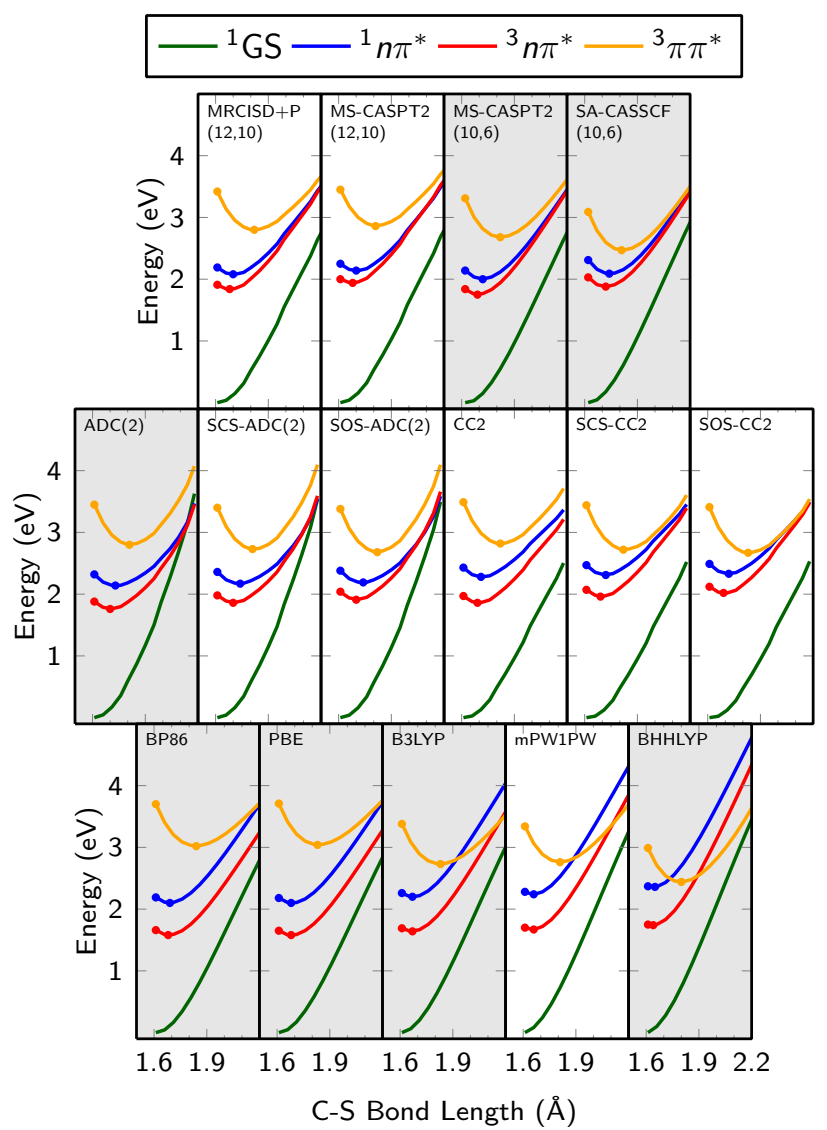

Figure 4: Potential energy scans for $\mathrm{CH}_{2} \mathrm{~S}$ along the $\mathrm{C}-\mathrm{S}$ bond length internal coordinate for different method and functional choices. The dots mark the FC point and excited-state minima. A shaded background indicates that the method was used in the nonadiabatic dynamics simulations. All coordinate systems use the same axis ranges.

exchange interaction of the involved electrons. The ${ }^{3} n \pi^{*}-{ }^{3} \pi \pi^{*}$ and ${ }^{1} n \pi^{*}-{ }^{3} \pi \pi^{*}$ gaps are larger, and fall below $0.2 \mathrm{eV}$ only above $2.1 \AA$; these gaps are related to the strength of the $\pi$ bond.

Among the other methods, only the MS-CASPT2 results with small basis set are fully consistent with the benchmark PES scans, with an RMSD $\Delta$ (RMSD of the ${ }^{1} n \pi^{*}-{ }^{3} n \pi^{*}$, ${ }^{1} n \pi^{*}-{ }^{3} \pi \pi^{*}$, and ${ }^{3} n \pi^{*}-{ }^{3} \pi \pi^{*}$ energy differences between 1.6 and $2.2 \AA$ ) of $0.02 \mathrm{eV}$. The relevant part of this PES scan is given by the C-S bond length evolution shown in Figure 3for MS-CASPT2, the maximum C-S bond length is about $2.1 \AA$. According to the potentials shown in Figure 4, at this bond length the $S_{1}-T_{2}$ energy gap is about $0.25 \mathrm{eV}$, whilst the SOC is only $170 \mathrm{~cm}^{-1}(0.021 \mathrm{eV})$, as given in Figure S3 in the SI. Thus, in this case the gap 
is still too large for ISC to occur in an efficient manner, and only the weak singlet-triplet mixing discussed above is observed.

According to the PES scan, ISC should only occur if the molecule is excited to at least $2.7 \mathrm{eV}$, which is the minimum energy of the ${ }^{3} \pi \pi^{*}$ state. At lower energies, only the ${ }^{1} n \pi^{*}$ state (minimum energy of $2.1 \mathrm{eV}$ ) can be populated, as ISC to the lower-energy ${ }^{3} n \pi^{*}$ state will not play a significant role due to the vanishing coupling between ${ }^{1} n \pi^{*}$ and ${ }^{3} n \pi^{*}$.

The SA-CASSCF method provides good qualitative shapes for the PESs, with smooth convergence of the energies of ${ }^{1} n \pi^{*},{ }^{3} n \pi^{*}$, and ${ }^{3} \pi \pi^{*}$ at long bond lengths. However, SACASSCF noticeably underestimates the energy of the ${ }^{3} \pi \pi^{*}$ and therefore obtains an RMSD $\Delta$ of $0.19 \mathrm{eV}$. The underestimated ${ }^{1} n \pi^{*}-{ }^{3} \pi \pi^{*}$ gap is also the reason why SA-CASSCF overestimates the ISC rate.

According to Figure 3, there is a significant number of the SA-CASSCF trajectories whose maximum bond length exceeds $2.1 \AA$ ( $10 \%$ of all trajectories), and some even go to $2.3 \AA$. Upon close inspection, these trajectories with long C-S bonds tend to undergo ISC more readily, although not all of them do so within the simulation time. This is consistent with the computed PESs, which show that at $2.1 \AA$ separation the $S_{1}-T_{2}$ energy gap is only $0.1 \mathrm{eV}$, with the SOCs being around $170 \mathrm{~cm}^{-1}(0.021 \mathrm{eV}$; see Figure S3 in the SI). The reduced gap compared to MS-CASPT2(10,6) - which had the same SOC value-provides a much higher probability for ISC at the $\operatorname{SA}-\operatorname{CASSCF}(10,6)$ level.

Regarding the computed PESs, the performance of the $\mathrm{ADC}(2)$ methods is no better than SA-CASSCF. These methods give comparable RMSD $\Delta \mathrm{s}$ : $0.20 \mathrm{eV}, 0.17 \mathrm{eV}$, and $0.19 \mathrm{eV}$ for regular, SCS-, and SOS-ADC(2). Furthermore,these methods show qualitative inaccuracies in the PESs. All ADC(2) variants predict a too steep $S_{0}$ PES (at MP2 level) at long bond lengths, which leads to the formation of a $S_{1} / S_{0}$ crossing at around $2.2 \AA$. The upshift of $S_{0}$ also increases the energy of ${ }^{3} \pi \pi^{*}$ and thus leads to large ${ }^{1} n \pi^{*}-{ }^{3} \pi \pi^{*}$ gaps, which never drop below $0.5 \mathrm{eV}$. This very large gap is the reason why $\mathrm{ADC}(2)$ predicts no ISC in the dynamics simulations, accidentally giving the correct qualitative result. The bad quality of 
the $\mathrm{ADC}(2)$ surfaces at long bond lengths can be linked to the very large values of the $\mathrm{D}_{1}$ and D2 diagnostics ${ }^{104,105}$ for the MP2 ground state, as presented in Figure S4 in the SI.

All CC2 methods provide similar RMSD $\Delta$ s of 0.18 eV (regular CC2), 0.23 eV (SCS-CC2), and $0.30 \mathrm{eV}$ (SOS-CC2). CC2 describes the ground state better than $\mathrm{ADC}(2)$, as CC2 obeys the non-crossing rule for $S_{0} / S_{1}$ while $\operatorname{ADC}(2)$ does not. ${ }^{106}$ This is unexpected, because at long bond distances the $\mathrm{D}_{1}$ and D2 diagnostics ${ }^{104,105}$ for the CC2 ground state (Figure S4 in the SI) reaches very high values. The energy gaps between the excited states are too large with CC2, whereas both SCS-CC2 and SOS-CC2 show too small ${ }^{1} n \pi^{*}-{ }^{3} \pi \pi^{*}$ gaps, apparently overcompensating from CC2. Judging from the PES scans, we expect that regular $\mathrm{CC} 2$ would give the same result as $\operatorname{ADC}(2)$, due to the large ${ }^{1} n \pi^{*}-{ }^{3} \pi \pi^{*}$ gap, whereas the SCS-CC2 and SOS-CC2 curves somewhat resemble the SA-CASSCF potentials and might give too large ISC rates.

As can be seen in Figure 4, all TDDFT methods fail at properly describing the PESs at stretched bond lengths, i.e., when the $\pi$ bond component of the C-S bond starts breaking. In particular, the $S_{0},{ }^{1} n \pi^{*}$, and ${ }^{3} n \pi^{*}$ PESs are too steep (the steeper the more HF exchange is employed, see below), and are nearly parallel in all cases, instead of converging towards each other. Due to this steepness, the ${ }^{1} n \pi^{*}$ and ${ }^{3} n \pi^{*}$ states also show minima at too short bond lengths. Conversely, the ${ }^{3} \pi \pi^{*}$ PES is actually rather good, especially with B3LYP and mPW1PW. However, this imbalance in the description of the states leads to wrong energy gaps. While the ${ }^{1} n \pi^{*}-{ }^{3} \pi \pi^{*}$ gaps are reasonably described by the GGA functionals - except for slightly too small gaps at large bond lengths - the functionals with HF exchange fail completely for the ${ }^{1} n \pi^{*}-{ }^{3} \pi \pi^{*}$ gap. Actually, for the three hybrid functionals (B3LYP with 20\% HF exchange, mPW1PW with 42.8\%, and BHHLYP with 50\%) at large bond lengths ${ }^{3} \pi \pi^{*}$ crosses with ${ }^{3} n \pi^{*}$, such that ${ }^{3} \pi \pi^{*}$ becomes the lowest triplet. This also means that the ${ }^{3} \pi \pi^{*}$ crosses with the ${ }^{1} n \pi^{*}$ state at very small bond lengths and at low energies. These inaccuracies will likely affect the ISC dynamics to a very large extent.

The results of the dynamics simulations for the TDDFT methods can fully be explained 


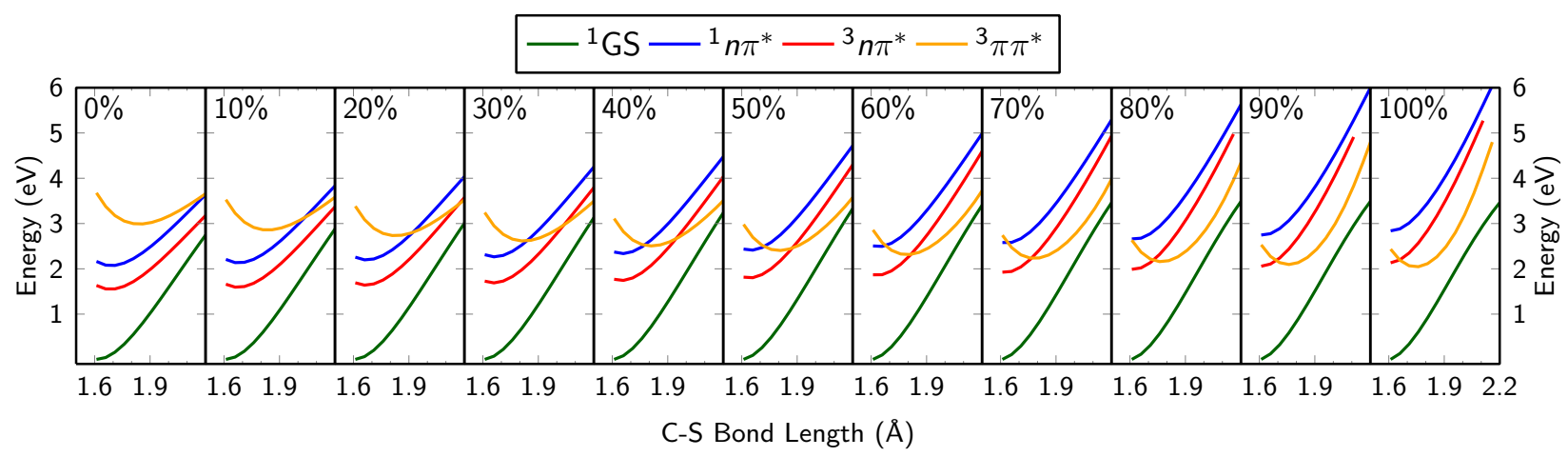

Figure 5: Potential energy scans for $\mathrm{CH}_{2} \mathrm{~S}$ along the $\mathrm{C}-\mathrm{S}$ bond length internal coordinate for different HF exchange \% in the B3LYP exchange-correlation functional.

by the shapes of the PESs. The GGA functionals show a maximum C-S bond length of about $2.0 \AA$ (in Figure 3), where the $S_{1}-T_{2}$ energy gap is still more than $0.3 \mathrm{eV}$. Consequently, no ISC was observed for these functionals. For B3LYP, the maximum C-S bond length is even smaller - only $1.95 \AA$ - but due to the $S_{1}-T_{2}$ crossing at around $1.95 \AA$, these short bond lengths are still sufficient to induce ISC. Finally, the BHHLYP functional shows the most complex C-S bond length evolution. For trajectories moving in the $S_{1}$ state (blue lines), the C-S bond length stays below $1.85 \AA$ due to the steep gradient of the PES. Nevertheless, the $S_{1}-T_{2}$ crossing is located at $1.75 \AA$, allowing for efficient ISC. In Figure 3 , the $T_{1}-T_{2}$ intersection seam (transition from gold to red) is also nicely visible at $1.9 \AA$. Compared to the other electronic structure methods, with BHHLYP the trajectories lose coherent C-S stretching much faster as the ensemble distributes over the three excited states.

\subsection{Influence of HF exchange}

As can be seen from Figure 4, the percentage of HF exchange has a strong influence on the shape of the PESs. In order to understand better this HF exchange dependence, we performed potential energy scans along the $\mathrm{C}-\mathrm{S}$ bond with the B3LYP functional, but varying the amount of HF exchange from zero to $100 \%$. The results are shown in Figure 5.

The variation of the amount of HF exchange leads to two clearly identifiable effects. First, at long bonds the ground state energy is increased, i.e., the ground state becomes steeper. 
The ${ }^{1} n \pi^{*}$ and ${ }^{3} n \pi^{*}$ states are similarly affected, although they are destabilized even more than the ground state. Hence, for larger HF exchange the states show even less convergent behavior than is already the case for GGA functionals ( $0 \% \mathrm{HF}$ exchange). The increase of steepness also move the ${ }^{1} n \pi^{*}$ and ${ }^{3} n \pi^{*}$ minima to higher energies and shorter bond lengths.

Second, the ${ }^{3} \pi \pi^{*}$ state is stabilized the more HF exchange is included, such that its minimum downshifts from $3 \mathrm{eV}$ to $2 \mathrm{eV}$; only at long bond lengths, its energy increases. The most dramatic effect of this downshift is the displacement of the ${ }^{1} n \pi^{*}-{ }^{3} \pi \pi^{*}$ crossing point, which is located around $2.2 \AA$ for $0 \%$ HF exchange, but at $1.7 \AA$ for $50 \%$ and below $1.6 \AA$ for more than $80 \%$. This is in stark contrast to the benchmark methods, where the two states never actually touch, but approach each other slowly $(0.2 \mathrm{eV}$ gap at around $2.1 \AA)$. Another effect of the downshift of the states is the formation of an accidental symmetry-allowed ${ }^{107}$ conical intersection between the ${ }^{3} \pi \pi^{*}$ state and the ${ }^{3} n \pi^{*}$ state which moves to smaller bond lengths and lower energies for larger HF exchange. While these two states do not touch for any of the wave function methods, with more than about $20 \%$ HF exchange in TDDFT they form an intersection which is located between $2.2 \AA$ (for $20 \% \mathrm{HF}$ exchange) and $1.7 \AA$ (at $100 \%)$.

In summary, Figure 5 shows that the amount of HF exchange is the underlying reason for the strong shifts in the PESs among the different DFT functionals. It can be expected that these shifts will markedly affect the ISC rate, as accessing the ${ }^{3} \pi \pi^{*}$ state from the ${ }^{1} n \pi^{*}$ state becomes increasingly easier due to the displacement of their crossing point. Similarly, these shifts will also enable faster internal conversion from the ${ }^{3} \pi \pi^{*}$ state to the ${ }^{3} n \pi^{*}$ state, due to the displacement of the corresponding conical intersection. This acceleration might be strongest for $50-70 \%$ HF exchange, because in this case the two crossings are very close to the state minima, such that only small C-S bond stretches are required. Hence, for systems with strongly stretched (double) bonds - like $\mathrm{CH}_{2} \mathrm{~S}$ - it appears that hybrid functionals should be avoided. Conversely, the GGA functionals with $0 \% \mathrm{HF}$ exchange seem to give reasonable PESs. 


\subsection{General Discussion}

Clearly, the inspection of the PESs along the most important vibrational mode provides significantly more insight into the performance of the electronic structure methods than the vertical excitation energies alone. Especially at stretched bond lengths, large differences between the electronic structure methods are found. It can be argued that a proper description of static correlation is required to correctly describe the stretching/breaking of the $\pi$ bond. Therefore, the single-reference/TDDFT methods struggle at long bond lengths, whereas they provide reasonable results at the FC geometry where static correlation is less important.

On the contrary, the SA-CASSCF potentials show that dynamic correlation is also required to obtain quantitatively correct results. Therefore, only the MS-CASPT2(10,6) level of theory fully agrees with the much more expensive benchmark computations and can be expected to deliver the right ISC dynamics for the right reasons.

Besides the shape of the PESs and in particular the $S_{1}-T_{2}$ gap, the ISC rate is also determined by the magnitude of the SOCs between these two states. A scan of the SOC norms for all methods is plotted in Figure S3 in the SI. This plot shows the SOCs between ${ }^{1} n \pi^{*}$ and ${ }^{3} \pi \pi^{*}$ for all methods, except for SCS/SOS-ADC(2) and the three CC2 variants, for which SOCs are currently not available in TuRBOmoLE. The other methods all yield qualitatively similar results for the SOCs, which was expected as SOCs do not strongly depend on electron correlation and the state characters are correct for all methods. ${ }^{108}$ Quantitatively, all multireference methods agree within $\pm 10 \mathrm{~cm}^{-1}$ with each other. $\mathrm{ADC}(2)$ underestimates the SOC elements by about $25 \mathrm{~cm}^{-1}$ (i.e., $127 \mathrm{~cm}^{-1}$ instead of $150-160 \mathrm{~cm}^{-1}$ at the FC geometry). The TDDFT methods underestimate the SOC elements by $10-15 \mathrm{~cm}^{-1}$. These results again show that the most difficult task for the electronic structure method is to produce accurate PESs.

From the above discussion of the PESs, it seems that the ISC propensity in the simulations is related to the difference between the ${ }^{1} n \pi^{*}$ vertical excitation energy and the ${ }^{3} \pi \pi^{*}$ minimum energy, as this difference controls how easy it is to reach the ${ }^{3} \pi \pi^{*}$ state. For the benchmark 
methods, this energy difference amounts to $0.61 \mathrm{eV}$. The differences for the well-performing methods are: $0.54 \mathrm{eV}$ for MS-CASPT2, $0.48 \mathrm{eV}$ for $\mathrm{ADC}(2), 0.83 \mathrm{eV}$ for BP86, and $0.86 \mathrm{eV}$ for PBE. The three failing methods have values of $0.47 \mathrm{eV}$ for B3LYP, $0.16 \mathrm{eV}$ for SACASSCF, and $0.07 \mathrm{eV}$ for BHHLYP. Clearly, a large energy difference leads to less ISC in the simulations. However, the fact that $\mathrm{ADC}(2)$ and B3LYP have almost the same value but still give very different ISC yields shows that this energy difference alone is not sufficient to predict the ISC dynamics. Instead, it is necessary to consider the actual shape of the PESs.

\section{Summary and Conclusions}

The vertical excitation energies and potential energy surfaces of the $S_{0}, S_{1}, T_{1}$, and $T_{2}$ states of $\mathrm{CH}_{2} \mathrm{~S}$ were investigated using 15 electronic structure methods to determine which of them are appropriate to describe the nonadiabatic dynamics within these states. Seven of these methods were selected to perform nonadiabatic dynamics simulations including intersystem crossing, employing the SHARC surface hopping method. The reference methods for the stationary calculations were the MRCISD $+\mathrm{P}(12,10)$ and MS-CASPT2 $(12,10)$ levels of theory, both using the ANO-RCC-VQZP basis set. The other considered methods were MS-CASPT2(10,6), SA-CASSCF(10,6), ADC(2) and CC2 (each with different flavors of spin-component scaling), and TDDFT with five different exchange-correlation functionals; these calculations employed double- $\zeta$ basis sets. In all cases, it was found that the vertical excitation energies at the Franck-Condon geometry for $S_{1}, T_{1}$, and $T_{2}$ are in reasonable agreement with the references and with available experimental values, suggesting at first glance that all methods should be able to properly describe the excited-state dynamics.

However, out of the seven methods employed in the dynamics simulations, only MSCASPT2(10,6), the GGA functionals BP86 and PBE as well as ADC(2) showed that no intersystem crossing should occur in this molecule on a sub-picosecond time scale. Important to note is that only MS-CASPT2 $(10,6)$ gave potentials virtually identical to the reference 
methods and thus provided the correct dynamics for the right reasons, whereas the latter three methods significantly overestimate the singlet-triplet gap. The remaining methodsSA-CASSCF(10,6), B3LYP, and BHHLYP - predicted a triplet yield between 3 and $21 \%$ within 500 fs, clearly wrong in light of the MS-CASPT2 results and experimental evidence.

Inspection of the excited-state potential energy surfaces showed that the good agreement at the Franck-Condon geometry is only deceptive, and that many of the electronic structure methods yield qualitatively wrong surfaces beyond the equilibrium geometry. Most prominently, the hybrid DFT functionals predicted unphysical state crossings at very low energies, whereas $\mathrm{ADC}(2)$ and GGA functionals overestimate the relevant singlet-triplet gaps. Thus, using the computed potential energy surfaces one can fully explain the variations in the results obtained in the dynamics simulations.

Overall, the present study illustrates how the results of nonadiabatic dynamics simulations are highly dependent on the choice of electronic structure method. Hence, for every such simulation it is crucial to validate the electronic structure method prior to the dynamics simulations. We caution that such a validation should not solely be carried out in the Franck-Condon region - as it is sometimes seen-because the fate of nonadiabatic dynamics is often decided far away from this region, where many electronic structure methods are less accurate. This was most visible in the case of B3LYP, which gives vertical excitation energies in close agreement with the references, but fails completely in the nonadiabatic dynamics predictions. The results also showcase that already errors of around $0.1-0.2 \mathrm{eV}$ in critical parts of the potential energy surfaces can lead to significant deviations in the results of the dynamics simulations. Certainly, the best option for any excited-state dynamics studies are correlated multi-reference methods, such as MRCI or MS-CASPT2, because only these methods can provide a truly balanced description of all parts of the potential energy surfaces, which is critical even in the seemingly simple molecule thioformaldehyde. However, as such methods are challenging or even unfeasible to employ on large molecules, a careful calibration of the electronic structure level of theory is decisive to obtain meaningful results. 


\section{Acknowledgement}

The Austrian Science Fund (FWF) within project M1815-N28 is acknowledged for funding. The computational results have been partially achieved using the Vienna Scientific Cluster (VSC), project 70880 .

\section{Supporting Information Available}

Active space orbitals, information on state characters at the Franck-Condon geometry, Spinorbit coupling surfaces, and results of excited-state optimizations.

\section{References}

(1) Chang, W.; Congreve, D. N.; Hontz, E.; Bahlke, M. E.; McMahon, D. P.; Reineke, S.; Wu, T. C.; Bulović, V.; Van Voorhis, T.; Baldo, M. A. Spin-dependent charge transfer state design rules in organic photovoltaics. Nat. Commun. 2015, 6, 6415.

(2) Adachi, C.; Baldo, M. A.; Thompson, M. E.; Forrest, S. R. Nearly $100 \%$ internal phosphorescence efficiency in an organic light-emitting device. J. Appl. Phys. 2001, $90,5048-5051$.

(3) Yang, X.; Neher, D.; Hertel, D.; Däubler, T. Highly Efficient Single-Layer Polymer Electrophosphorescent Devices. Adv. Mater. 2004, 16, 161-166.

(4) Massey, A.; Xu, Y.-Z.; Karran, P. Photoactivation of DNA Thiobases as a Potential Novel Therapeutic Option. Curr. Biol. 2001, 11, 1142-1146.

(5) Dolmans, D. E. J. G. J.; Fukumura, D.; Jain, R. K. Photodynamic therapy for cancer. Nat. Rev. Cancer 2003, 3, 380. 
(6) Chergui, M. On the interplay between charge, spin and structural dynamics in transition metal complexes. Dalton Trans. 2012, 41, 13022-13029.

(7) Richter, M.; Marquetand, P.; González-Vázquez, J.; Sola, I.; González, L. SHARC: Ab Initio Molecular Dynamics with Surface Hopping in the Adiabatic Representation Including Arbitrary Couplings. J. Chem. Theory Comput. 2011, 7, 1253-1258.

(8) Mai, S.; Marquetand, P.; González, L. A General Method to Describe Intersystem Crossing Dynamics in Trajectory Surface Hopping. Int. J. Quantum Chem. 2015, $115,1215-1231$.

(9) Mai, S.; Marquetand, P.; González, L. Nonadiabatic dynamics: The SHARC approach. WIREs Comput. Mol. Sci. 2018, 8, e1370.

(10) Granucci, G.; Persico, M.; Spighi, G. Surface hopping trajectory simulations with spin-orbit and dynamical couplings. J. Chem. Phys. 2012, 13\%, 22 A501.

(11) Franco de Carvalho, F.; Tavernelli, I. Nonadiabatic dynamics with intersystem crossings: A time-dependent density functional theory implementation. J. Chem. Phys. 2015, 143, 224105.

(12) Pederzoli, M.; Pittner, J. A new approach to molecular dynamics with non-adiabatic and spin-orbit effects with applications to $\mathrm{QM} / \mathrm{MM}$ simulations of thiophene and selenophene. J. Chem. Phys. 2017, 146, 114101.

(13) Wiggins, P.; Williams, J. A. G.; Tozer, D. J. Excited state surfaces in density functional theory: A new twist on an old problem. J. Chem. Phys. 2009, 131, 091101.

(14) Gozem, S.; Huntress, M.; Schapiro, I.; Lindh, R.; Granovsky, A. A.; Angeli, C.; Olivucci, M. Dynamic Electron Correlation Effects on the Ground State Potential Energy Surface of a Retinal Chromophore Model. J. Chem. Theory Comput. 2012, 8, 4069-4080. 
(15) Barbatti, M.; Lan, Z.; Crespo-Otero, R.; Szymczak, J. J.; Lischka, H.; Thiel, W. Critical Appraisal of Excited State Nonadiabatic Dynamics Simulations of 9H-Adenine. J. Chem. Phys. 2012, 137, 22A503.

(16) Valsson, O.; Filippi, C.; Casida, M. E. Regarding the use and misuse of retinal protonated Schiff base photochemistry as a test case for time-dependent density-functional theory. J. Chem. Phys. 2015, 142, 144104.

(17) Steer, R. P. Structure and decay dynamics of electronic excited states of thiocarbonyl compounds. Rev. Chem. Intermed. 1981, 4, 1-41.

(18) Maciejewski, A.; Steer, R. P. The photophysics, physical photochemistry, and related spectroscopy of thiocarbonyls. Chem. Rev. 1993, 93, 67-98.

(19) Clouthier, D. J.; Ramsay, D. A. The Spectroscopy of Formaldehyde and Thioformaldehyde. Annu. Rev. Phys. Chem. 1983, 34, 31-58.

(20) Clouthier, D. J.; Moule, D. C. Relationships and Mechanisms in the Periodic Table; Springer Berlin Heidelberg: Berlin, Heidelberg, 1989; pp 167-247.

(21) Grein, F.; Hachey, M. R. J. The $\pi, \pi^{*}$ state in formaldehyde and thioformaldehyde. Int. J. Quantum Chem. 1996, 60, 1661-1671.

(22) Kawasaki, M.; Kasatani, K.; Ogawa, Y.; Sato, H. Spectra and emission lifetimes of $\mathrm{H}_{2} \mathrm{CS}\left(\tilde{A}^{1} A_{2}\right)$. Chem. Phys. 1983, 74, $83-88$.

(23) Moule, D. C.; Lim, E. C. Highly Varying Photophysical Properties of Thiocarbonyls: Validation of Fundamental Theoretical Concepts of Electronic Radiationless Transitions. J. Phys. Chem. A 2002, 106, 3072-3076.

(24) Fu, B.; Shepler, B. C.; Bowman, J. M. Three-State Trajectory Surface Hopping Studies of the Photodissociation Dynamics of Formaldehyde on ab Initio Potential Energy Surfaces. J. Am. Chem. Soc. 2011, 133, 7957-7968. 
(25) Lischka, H.; Shepard, R.; Shavitt, I.; Pitzer, R. M.; Dallos, M.; Müller, T.; Szalay, P. G.; Brown, F. B.; Ahlrichs, R.; Böhm, H. J.; Chang, A.; Comeau, D. C.; Gdanitz, R.; Dachsel, H.; Ehrhardt, C.; Ernzerhof, M.; Höchtl, P.; Irle, S.; Kedziora, G.; Kovar, T.; Parasuk, V.; Pepper, M. J. M.; Scharf, P.; Schiffer, H.; Schindler, M.; Schüler, M.; Seth, M.; Stahlberg, E. A.; Zhao, J.-G.; Yabushita, S.; Zhang, Z.; Barbatti, M.; Matsika, S.; Schuurmann, M.; Yarkony, D. R.; Brozell, S. R.; Beck, E. V.; ; Blaudeau, J.-P.; Ruckenbauer, M.; Sellner, B.; Plasser, F.; Szymczak, J. J. COLUMBUS, an ab initio electronic structure program, release 7.0. 2012.

(26) Lischka, H.; Müller, T.; Szalay, P. G.; Shavitt, I.; Pitzer, R. M.; Shepard, R. Columbus - a program system for advanced multireference theory calculations. WIREs Comput. Mol. Sci. 2011, 1, 191-199.

(27) Lischka, H.; Shepard, R.; Pitzer, R. M.; Shavitt, I.; Dallos, M.; Müller, T.; Szalay, P. G.; Seth, M.; Kedziora, G. S.; Yabushita, S.; Zhang, Z. High-level multireference methods in the quantum-chemistry program system COLUMBUS: Analytic MR-CISD and MR-AQCC gradients and MR-AQCC-LRT for excited states, GUGA spin-orbit CI and parallel CI density. Phys. Chem. Chem. Phys. 2001, 3, 664-673.

(28) Pople, J. A.; Seeger, R.; Krishnan, R. Variational configuration interaction methods and comparison with perturbation theory. Int. J. Quantum Chem. 1977, 12, 149-163.

(29) Roos, B. O.; Lindh, R.; Malmqvist, P.-Å.; Veryazov, V.; Widmark, P.-O. New Relativistic ANO Basis Sets for Transition Metal Atoms. J. Phys. Chem. A 2005, 109, $6575-6579$.

(30) Aquilante, F.; Autschbach, J.; Carlson, R. K.; Chibotaru, L. F.; Delcey, M. G.; De Vico, L.; Fdez. Galván, I.; Ferré, N.; Frutos, L. M.; Gagliardi, L.; Garavelli, M.; Giussani, A.; Hoyer, C. E.; Li Manni, G.; Lischka, H.; Ma, D.; Malmqvist, P.A.; Müller, T.; Nenov, A.; Olivucci, M.; Pedersen, T. B.; Peng, D.; Plasser, F.; 
Pritchard, B.; Reiher, M.; Rivalta, I.; Schapiro, I.; Segarra-Martí, J.; Stenrup, M.; Truhlar, D. G.; Ungur, L.; Valentini, A.; Vancoillie, S.; Veryazov, V.; Vysotskiy, V. P.; Weingart, O.; Zapata, F.; Lindh, R. Molcas 8: New Capabilities for Multiconfigurational Quantum Chemical Calculations Across the Periodic Table. J. Comput. Chem. 2015, 37, 506-541.

(31) Andersson, K.; Malmqvist, P.-A.; Roos, B. O.; Sadlej, A. J.; Wolinski, K. Secondorder perturbation theory with a CASSCF reference function. J. Phys. Chem. 1990, $94,5483-5488$.

(32) Andersson, K.; Malmqvist, P.-A.; Roos, B. O. Second-order perturbation theory with a complete active space self-consistent field reference function. J. Chem. Phys. 1992, $96,1218-1226$.

(33) Finley, J.; Malmqvist, P.-A.; Roos, B. O.; Serrano-Andrés, L. The Multi-State CASPT2 Method. Chem. Phys. Lett. 1998, 288, 299-306.

(34) Aquilante, F.; Malmqvist, P.-Å.; Pedersen, T. B.; Ghosh, A.; Roos, B. O. Cholesky Decomposition-Based Multiconfiguration Second-Order Perturbation Theory (CDCASPT2): Application to the Spin-State Energetics of CoIII(diiminato)(NPh). J. Chem. Theory Comput. 2008, 4, 694-702.

(35) Dunning, T. H. Gaussian Basis Sets for Use in Correlated Molecular Calculations. I. The Atoms Boron Through Neon and Hydrogen. J. Chem. Phys. 1989, 90, 1007-1023.

(36) Kendall, R. A.; Dunning Jr., T. H.; Harrison, R. J. Electron affinities of the first-row atoms revisited. Systematic basis sets and wave functions. J. Chem. Phys. 1992, 96, $6796-6806$.

(37) Woon, D. E.; Dunning, T. H. Gaussian basis sets for use in correlated molecular calculations. IV. Calculation of static electrical response properties. J. Chem. Phys. 1994, 100, 2975-2988. 
(38) Olsen, J. The CASSCF method: A perspective and commentary. Int. J. Quant. Chem. 2011, 111, 3267-3272.

(39) TURBOMOLE V6.4 2012, a development of University of Karlsruhe and Forschungszentrum Karlsruhe GmbH, 1989-2007, TURBOMOLE GmbH, since 2007; available from http://www.turbomole.com.

(40) Schirmer, J. Beyond the random-phase approximation: A new approximation scheme for the polarization propagator. Phys. Rev. A 1982, 26, 2395-2416.

(41) Hättig, C. Structure Optimizations for Excited States with Correlated Second-Order Methods: CC2 and ADC(2). Adv. Quant. Chem. 2005, 50, 37-60.

(42) Weigend, F.; Ahlrichs, R. Balanced basis sets of split valence, triple zeta valence and quadruple zeta valence quality for H to Rn: Design and assessment of accuracy. Phys. Chem. Chem. Phys. 2005, 7, 3297-3305.

(43) Christiansen, O.; Koch, H.; Jørgensen, P. The second-order approximate coupled cluster singles and doubles model CC2. Chem. Phys. Lett. 1995, 243, $409-418$.

(44) Christiansen, O.; Jørgensen, P.; Hättig, C. Response functions from Fourier component variational perturbation theory applied to a time-averaged quasienergy. Int. J. Quantum Chem. 1998, 68, 1-52.

(45) Hellweg, A.; Grün, S. A.; Hättig, C. Benchmarking the performance of spin-component scaled CC2 in ground and electronically excited states. Phys. Chem. Chem. Phys. 2008, 10, 4119-4127.

(46) Fonseca Guerra, C.; Snijders, G. J.; te Velde, G.; Baerends, J. E. Towards an order-N DFT method. Theor. Chem. Acc. 99, 391-403. 
(47) te Velde, G.; Bickelhaupt, F. M.; Baerends, E. J.; Fonseca Guerra, C.; van Gisbergen, S. J. A.; Snijders, J. G.; Ziegler, T. Chemistry with ADF. J. Comput. Chem. 2001, 22, 931-967.

(48) ADF2014 (locally modified), SCM, Theoretical Chemistry, Vrije Universiteit, Amsterdam, The Netherlands, http://www.scm.com. Program.

(49) Becke, A. D. Density-functional exchange-energy approximation with correct asymptotic behavior. Phys. Rev. A 1988, 38, 3098-3100.

(50) Perdew, J. P. Density-functional approximation for the correlation energy of the inhomogeneous electron gas. Phys. Rev. B 1986, 33, 8822-8824.

(51) van Lenthe, E.; Baerends, E. J. Optimized Slater-type basis sets for the elements 1-118. J. Comput. Chem. 2003, 24, 1142-1156.

(52) Perdew, J. P.; Burke, K.; Ernzerhof, M. Generalized Gradient Approximation Made Simple. Phys. Rev. Lett. 1996, 77, 3865-3868.

(53) Becke, A. D. Density-functional thermochemistry. III. The role of exact exchange. J. Chem. Phys. 1993, 98, 5648-5652.

(54) Lee, C.; Yang, W.; Parr, R. G. Development of the Colle-Salvetti correlation-energy formula into a functional of the electron density. Phys. Rev. B 1988, 37, 785-789.

(55) Stephens, P. J.; Devlin, F. J.; Chabalowski, C. F.; Frisch, M. J. Ab Initio Calculation of Vibrational Absorption and Circular Dichroism Spectra Using Density Functional Force Fields. J. Phys. Chem. 1994, 98, 11623-11627.

(56) Adamo, C.; Barone, V. Exchange functionals with improved long-range behavior and adiabatic connection methods without adjustable parameters: The mPW and mPW1PW models. J. Chem. Phys. 1998, 108, 664-675. 
(57) Becke, A. D. A new mixing of Hartree-Fock and local density-functional theories. J. Chem. Phys. 1993, 98, 1372-1377.

(58) Werner, H.-J.; Knowles, P. J.; Knizia, G.; Manby, F. R.; Schütz, M.; Celani, P.; Korona, T.; Lindh, R.; Mitrushenkov, A.; Rauhut, G.; Shamasundar, K. R.; Adler, T. B.; Bernhardsson, R. D. A. A.; Berning, A.; Cooper, D. L.; Deegan, M. J. O.; Dobbyn, A. J.; Eckert, F.; Goll, E.; Hampel, C.; Hesselmann, A.; Hetzer, G.; Hrenar, T.; Jansen, G.; Köppland, C.; Liu, Y.; Lloyd, A. W.; Mata, R. A.; May, A. J.; J.McNicholas, S.; Meyer, W.; Mura, M. E.; Nicklass, A.; P.O’Neill, D.; Palmieri, P.; Peng, D.; Pflüger, K.; Pitzerand, R.; Reiher, M.; Shiozaki, T.; Stoll, H.; Stone, A. J.; R.Tarroni,; Thorsteinsson, T.; Wang, M. MOLPRO, version 2012.1, a package of ab initio programs. 2012; see https://www.molpro.net/.

(59) Werner, H.-J.; Knowles, P. J.; Knizia, G.; Manby, F. R.; Schütz, M. Molpro: A generalpurpose quantum chemistry program package. WIREs Comput. Mol. Sci. 2012, 2, 242-253.

(60) Hampel, C.; Peterson, K. A.; Werner, H.-J. A comparison of the efficiency and accuracy of the quadratic configuration interaction (QCISD), coupled cluster (CCSD), and Brueckner coupled cluster (BCCD) methods. Chem. Phys. Lett. 1992, 190, 1-12.

(61) Hrenar, T.; Rauhut, G.; Werner, H.-J. Impact of Local and Density Fitting Approximations on Harmonic Vibrational Frequencies. J. Phys. Chem. A 2006, 110, 20602064.

(62) Richter, M.; Marquetand, P.; González-Vázquez, J.; Sola, I.; González, L. Femtosecond Intersystem Crossing in the DNA Nucleobase Cytosine. J. Phys. Chem. Lett. 2012, 3, 3090-3095.

(63) Mai, S.; Marquetand, P.; González, L. Intersystem Crossing Pathways in the Non- 
canonical Nucleobase 2-Thiouracil: A Time-Dependent Picture. J. Phys. Chem. Lett. 2016, 7, 1978-1983.

(64) Mai, S.; Marquetand, P.; González, L. Non-Adiabatic Dynamics in $\mathrm{SO}_{2}$ : II. The Role of Triplet States Studied by Surface-Hopping Simulations. J. Chem. Phys. 2014, 140, 204302.

(65) Casida, M.; Huix-Rotllant, M. Progress in Time-Dependent Density-Functional Theory. Annu. Rev. Phys. Chem. 2012, 63, 287-323.

(66) Atkins, A. J.; González, L. Trajectory Surface-Hopping Dynamics Including Intersystem Crossing in $\left[\mathrm{Ru}(\mathrm{bpy})_{3}\right]^{2+}$. J. Phys. Chem. Lett. 2017, 8, 3840-3845.

(67) Mai, S.; Plasser, F.; Pabst, M.; Neese, F.; Köhn, A.; González, L. Surface Hopping Dynamics Including Intersystem Crossing using the Algebraic Diagrammatic Construction Method. J. Chem. Phys. 2017, 147, 184109.

(68) Nakajima, T.; Hirao, K. The Douglas-Kroll-Hess Approach. Chem. Rev. 2012, 112, 385-402.

(69) Zobel, J. P.; Nogueira, J. J.; González, L. The IPEA Dilemma in CASPT2. Chem. Sci. 2017, 8, 1482-1499.

(70) Ghigo, G.; Roos, B. O.; Malmqvist, P.-Å. A Modified Definition of the Zeroth-Order Hamiltonian in Multiconfigurational Perturbation Theory (CASPT2). Chem. Phys. Lett. 2004, 396, 142-149.

(71) Forsberg, N.; Malmqvist, P.-Å. Multiconfiguration Perturbation Theory with Imaginary Level Shift. Chem. Phys. Lett. 1997, 274, 196-204.

(72) Hättig, C.; Weigend, F. CC2 excitation energy calculations on large molecules using the resolution of the identity approximation. J. Chem. Phys. 2000, 113, 5154-5161. 
(73) van Lenthe, E.; Ehlers, A.; Baerends, E. J. Geometry optimizations in the zero order regular approximation for relativistic effects. J. Chem. Phys. 1999, 110, 8943-8953.

(74) van Lenthe, E.; Baerends, E. J.; Snijders, J. G. Relativistic regular two-component Hamiltonians. J. Chem. Phys. 1993, 99, 4597-4610.

(75) van Lenthe, E.; Baerends, E. J.; Snijders, J. G. Relativistic total energy using regular approximations. J. Chem. Phys. 1994, 101, 9783-9792.

(76) van Lenthe, E.; Snijders, J. G.; Baerends, E. J. The zero-order regular approximation for relativistic effects: The effect of spin-orbit coupling in closed shell molecules. $J$. Chem. Phys. 1996, 105, 6505-6516.

(77) van Lenthe, E.; van Leeuwen, R.; Baerends, E. J.; Snijders, J. G. Relativistic regular two-component Hamiltonians. Int. J. Quantum Chem. 1996, 57, 281-293.

(78) Becke, A. D. A multicenter numerical integration scheme for polyatomic molecules. $J$. Chem. Phys. 1988, 88, 2547-2553.

(79) Franchini, M.; Philipsen, P. H. T.; Visscher, L. The Becke Fuzzy Cells Integration Scheme in the Amsterdam Density Functional Program Suite. J. Comput. Chem. 2013, 34, 1819-1827.

(80) Franchini, M.; Philipsen, P. H. T.; van Lenthe, E.; Visscher, L. Accurate Coulomb Potentials for Periodic and Molecular Systems through Density Fitting. J. Chem. Theory Comput. 2014, 10, 1994-2004.

(81) Hirata, S.; Head-Gordon, M. Time-dependent density functional theory within the Tamm-Dancoff approximation. Chem. Phys. Lett. 1999, 314, $291-299$.

(82) Hirata, S.; Head-Gordon, M. Time-dependent density functional theory for radicals: An improved description of excited states with substantial double excitation character. Chem. Phys. Lett. 1999, 302, 375-382. 
(83) Maitra, N. T.; Zhang, F.; Cave, R. J.; Burke, K. Double excitations within timedependent density functional theory linear response. J. Chem. Phys. 2004, 120, 59325937.

(84) Yabushita, S.; Zhang, Z.; Pitzer, R. M. Spin-Orbit Configuration Interaction Using the Graphical Unitary Group Approach and Relativistic Core Potential and Spin-Orbit Operators. J. Phys. Chem. A 1999, 103, 5791-5800.

(85) Mai, S.; Müller, T.; Marquetand, P.; Plasser, F.; Lischka, H.; González, L. Perturbational Treatment of Spin-Orbit Coupling for Generally Applicable High-Level MultiReference Methods. J. Chem. Phys. 2014, 141, 074105.

(86) Malmqvist, P.-Å.; Roos, B. O.; Schimmelpfennig, B. The restricted active space (RAS) state interaction approach with spin-orbit coupling. Chem. Phys. Lett. 2002, 357, $230-240$.

(87) Pabst, M.; Köhn, A. Implementation of transition moments between excited states in the approximate coupled-cluster singles and doubles model. J. Chem. Phys. 2008, $129,214101$.

(88) Wang, F.; Ziegler, T.; van Lenthe, E.; van Gisbergen, S.; Baerends, E. J. The calculation of excitation energies based on the relativistic two-component zeroth-order regular approximation and time-dependent density-functional with full use of symmetry. J. Chem. Phys. 2005, 122, 204103.

(89) Wang, F.; Ziegler, T. A simplified relativistic time-dependent density-functional theory formalism for the calculations of excitation energies including spin-orbit coupling effect. J. Chem. Phys. 2005, 123, 154102.

(90) Schinke, R. Photodissociation Dynamics: Spectroscopy and Fragmentation of Small Polyatomic Molecules; Cambridge University Press, 1995. 
(91) Dahl, J. P.; Springborg, M. The Morse oscillator in position space, momentum space, and phase space. J. Chem. Phys. 1988, 88, 4535-4547.

(92) Crespo-Otero, R.; Barbatti, M. Spectrum simulation and decomposition with nuclear ensemble: formal derivation and application to benzene, furan and 2-phenylfuran. Theor. Chem. Acc. 2012, 131, 1237.

(93) Granucci, G.; Persico, M.; Toniolo, A. Direct Semiclassical Simulation of Photochemical Processes with Semiempirical Wave Functions. J. Chem. Phys. 2001, 114, 1060810615.

(94) Plasser, F.; Ruckenbauer, M.; Mai, S.; Oppel, M.; Marquetand, P.; González, L. Efficient and Flexible Computation of Many-Electron Wave Function Overlaps. J. Chem. Theory Comput. 2016, 12, 1207.

(95) Plasser, F.; Crespo-Otero, R.; Pederzoli, M.; Pittner, J.; Lischka, H.; Barbatti, M. Surface Hopping Dynamics with Correlated Single-Reference Methods: 9H-Adenine as a Case Study. J. Chem. Theory Comput. 2014, 10, 1395-1405.

(96) Tapavicza, E.; Tavernelli, I.; Rothlisberger, U. Trajectory Surface Hopping within Linear Response Time-Dependent Density-Functional Theory. Phys. Rev. Lett. 2007, 98, 023001 .

(97) Casida, M. E. Recent Advances in Density Functional Methods Part I; World Scientific: Singapore, 1995; pp 155-192.

(98) Granucci, G.; Persico, M. Critical appraisal of the fewest switches algorithm for surface hopping. J. Chem. Phys. 2007, 126, 134114.

(99) González, L.; Escudero, D.; Serrano-Andrés, L. Progress and Challenges in the Calculation of Electronic Excited States. ChemPhysChem 2012, 13, 28-51. 
(100) Peach, M. J. G.; Tozer, D. J. Overcoming Low Orbital Overlap and Triplet Instability Problems in TDDFT. J. Phys. Chem. A 2012, 116, 9783-9789.

(101) Englman, R.; Jortner, J. The energy gap law for radiationless transitions in large molecules. Mol. Phys. 1970, 18, 145-164.

(102) Clouthier, D. J.; Ramsay, D. A.; Birss, F. W. Singlet-triplet perturbations in the $\tilde{A}$ ${ }^{1} \mathrm{~A}_{2}(\nu=0)$ state of thioformaldehyde. J. Chem. Phys. 1983, 79, 5851-5862.

(103) Curchod, B. F. E.; Rauer, C.; Marquetand, P.; González, L.; Martínez, T. J. Communication: GAIMS - Generalized Ab Initio Multiple Spawning for both internal conversion and intersystem crossing processes. J. Chem. Phys. 2016, 144, 101102.

(104) Janssen, C. L.; Nielsen, I. M. New diagnostics for coupled-cluster and Møller-Plesset perturbation theory. Chem. Phys. Lett. 1998, 290, 423-430.

(105) Nielsen, I. M.; Janssen, C. L. Double-substitution-based diagnostics for coupled-cluster and Møller-Plesset perturbation theory. Chem. Phys. Lett. 1999, 310, 568-576.

(106) Tuna, D.; Lefrancois, D.; Wolański, Ł.; Gozem, S.; Schapiro, I.; Andruniów, T.; Dreuw, A.; Olivucci, M. Assessment of Approximate Coupled-Cluster and AlgebraicDiagrammatic-Construction Methods for Ground- and Excited-State Reaction Paths and the Conical-Intersection Seam of a Retinal-Chromophore Model. J. Chem. Theory Comput. 2015, 11, 5758-5781.

(107) Domcke, W., Yarkony, D. R., Köppel, H., Eds. Conical Intersections: Electronic Structure, Dynamics and Spectroscopy; World Scientific Publishing, 2004.

(108) Jovanović, V.; Lyskov, I.; Kleinschmidt, M.; Marian, C. M. On the performance of DFT/MRCI-R and MR-MP2 in spin-orbit coupling calculations on diatomics and polyatomic organic molecules. Mol. Phys. 2017, 115, 109-137. 


\section{Grahical TOC Entry}
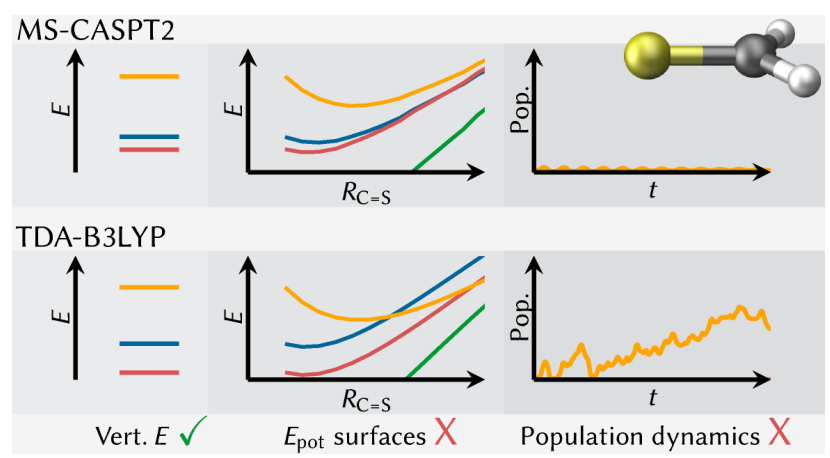